\title{
An Updated Review on the Role of Physical Activity and Nutrition in the Treatment of Renal Diseases
}

\author{
Mahsa Rastegar M M ${ }^{1}$, Mohammadreza Mohseni ${ }^{2}$ and Sivakumar J T Gowder ${ }^{3,4 *}$ \\ ${ }^{1}$ Faculty of Sports Sciences, Hakim Sabzevari University, Iran \\ ${ }^{2}$ Lifestyle Gym, Tehran, Iran \\ ${ }^{3}$ Department for Management of Science and Technology Development, Ton Duc Thang University, Ho Chi Minh City, Vietnam \\ ${ }^{4}$ Faculty of Applied Sciences, Ton Duc Thang University, Ho Chi Minh City, Vietnam \\ *Corresponding author: Sivakumar J T Gowder, Ton Duc Thang University, Ho Chi Minh City, Vietnam. \\ Email: sivakumargowder@tdtu.edu.vn
}

ARTICLE INFO

Received: 慧 March 06, 2020

Published: March 16, 2020

Citation: Mahsa Rastegar M M, Mohammadreza M, Sivakumar J T Gowder. "An Updated Review on the Role of Physical Activity and Nutrition in the Treatment of Renal Diseases" Biomed J Sci \& Tech Res 26(4)2020. BJSTR. MS.ID.004380.

Keywords: Chronic Kidney Disease; End Stage Renal Disease; Sedentary Behavior; Physical Activity; Nutrition; Glomerular Filtration Rate; Cognitive impairment; Parenchyma; Proteinuria, Leukoaraiosis; Homeostasis; Renal cell carcinoma; Ameliorate dialysis-related symptoms

\begin{abstract}
Kidney diseases are divided into two: acute and chronic sub-groups that lasted less and more than three months, respectively. Chronic Kidney Diseases which is known as CKD is a gradual kidney dysfunction that leads to End-Stage Renal Disease (ESRD), a condition that kidney stops to work properly and needs to be treated by transplant or dialysis. ESRD accompanies with reduced Glomerular Filtration Rate (GFR) that according to reduction of kidney filtration rate, it is classified into five stages. Kidney damages resulted in various direct and indirect dysfunctions, such as proteinuria, albuminuria, metabolic acidosis or even stroke, white matter diseases, intracerebral microbleeds and cognitive impairment. Sedentary lifestyle is one of the key effective factors on epidemic of chronic diseases such CKD and ESRD. CKD patients normally have low levels of physical activity compare to their same age groups, that results in cardiovascular dysfunction, physical function deterioration, and kidney diseases. Performing of active lifestyle and doing of exercise plus intake of appropriate diet could control CKD-related disorders such as glomerular disorders, metabolic acidosis, anemia or inflammation and could rearrange balance of acid-base status of kidney and make tempos of kidney diseases progression slow in an appropriate manner.
\end{abstract}

Abbrevations: CKD: Chronic Kidney Diseases; ESRD: End-Stage Renal Disease; GFR: Glomerular Filtration Rate; VLDLs: Very-Low-Density Lipoproteins; IDLs: IntermediateDensity Lipoproteins; HDL: High-Density Lipoproteins; EVs: Extracellular Vehicles; ANS: Autonomic Nervous System; UPS: Ubiquitin-Proteasome System; IGF-1: Insulin-Like Growth Factor 1; PRAL: Potential Renal Acid Load; apoE: Apolipoprotein E

\section{Introduction}

Kidney-related diseases are those disorders which a person's kidneys are damaged and disable to work properly, which means that wastes are built up in the body. Most kidney diseases are invisible as they do not have symptoms until they become chronic and in this stage their outcomes are irreversible, and treatments could just slow the progression of disease. Damage to parenchyma as functional part of the kidney that includes the renal cortex, large blood vessels, or even collecting duct system are those kidney damages that could be calculated by respective markers rather than direct kidney examination. Kidney diseases cause vast majority of secondary problems such as stroke, white matter diseases, intracerebral microbleeds and cognitive impairment [1-3].

\section{Kidney Diseases}

Acute Kidney Injury (AKI) is a sudden and temporary kidney's dysfunction such as acute Glomerulo Nephritis (GN) which is inflammation of the glomeruli and decrease of Glomerular Filtration Rate (GFR) and is showed by serum creatinine elevation but 
creatinine is a delayed serum marker that does not accurately reflect the GFR in renal function changes [4]. AKI has different etiologies which is not the priority of this review article. In contrast, Chronic Kidney Disease (CKD) which is lasted more than three months with implications for health, is gradual kidney dysfunction and lead to renal disease or End-Stage Renal Disease (ESRD), a condition that kidney fails and needs to be treated by transplant or dialysis and could be a risk factor for renal cell carcinoma [1, 5]. CKD has totally five stages. In stage one, there is kidney damage, but its function is normal, that is, GFR is estimated $\geq 90 \mathrm{~mL} / \mathrm{min}$ per $1.73 \mathrm{~m} 2$. GFR is the rate at which the kidneys filter wastes and extra fluid from the blood which in normal body is about 90 to $120 \mathrm{~mL} / \mathrm{min} / 1.73 \mathrm{~m} 2$. The CKD-EPI equation, expressed as a single equation, in stage one of GFR, persistent proteinuria is existed.

Proteinuria is a term to define abnormal loss of plasma proteins due to some reasons such as increase glomerular permeability to those proteins that should not be lost, that is called glomerular proteinuria, incomplete tubular reabsorption, that is called tubular proteinuria, and increase plasma concentration of low-molecularweight proteins that is called overproduction proteinuria [1]. In stage two of CKD, kidney is damaged, and their functions will loss mildly. GFR reaches to $60-89 \mathrm{~mL} / \mathrm{min}$ per $1.73 \mathrm{~m} 2$ with proteinuria. According to experimental studies, proteinuria plays a vital role in pathogenesis of CKD. In stages three to five, there would be no proteinuria and the function of kidney damage from mild-severe to kidney failure with GFR from about $40 \mathrm{~mL} / \mathrm{min}$ per $1.73 \mathrm{~m} 2$ to less than $15 \mathrm{~mL} / \mathrm{min}$ per $1.73 \mathrm{~m} 2$ [1,2,6]. Another form of kidney damage is albuminuria. In albuminuria, the individual's loss too much albumin in their urine. Albumin is a protein which is made by liver and helps keep fluid in blood stream, and it carries different substances such as hormones and vitamins. Albuminuria is an earlier marker in glomerular diseases and often associated with hypertension and obesity [1]. Uremia is also another complication of CKD in which the kidney could not get rid of urea and other waste products which build up in body and their accumulation lead to intoxication.

Acute uremia is mostly recognized by seizures and coma, while chronic progressive uremia resulted in peripheral neuropathy and encephalopathy. Peripheral nerves make complex network that connect brain and spinal cord to the rest of the body which in case of peripheral neuropathy, this connection results in damage. In encephalopathy, patient faces broad changes from mild memory loss to even coma and death. Renal disease cause cerebrovascular and cardiovascular complications which are a kind of link between renal and neurological diseases $[3,4]$. Stroke is one of the foremost cardiovascular causes of death in CKD and ESRD, especially in dialysis patients, and mortality rate is also high which may be due to poor hypertension management of these patients; and is greater in women than in men. There are other ESRD-induced cerebrovascular diseases such as leukoaraiosis (pathological appearance of the brain white matter near the lateral ventricles due to perfusion disturbances within the arterioles), silent cerebral infarct that does not cause any symptoms but still damage brain tissue which appear as weakness or paralysis, and cerebral microbleeds, as chronic accumulation of small blood products in brain which per se encourage dementia and even increase mortality rate. It is suggested that ESRD indirectly increase cerebrovascular disease (e.g. stroke) due to dialysis-related factors such as blood pressure fluctuations [3].

Glomerular disorders are one of the CKD complications. To clarify glomerular disorders, at first, we need to evaluate lipid nephrotoxicity. Lipid nephrotoxicity was defined as glomerulosclerosis due to hyperlipidemia. Apolipoprotein E (apoE) is a protein involved in the metabolism of fats in the body. This protein is a lipid transporter and binds with fats (lipids) and is responsible for packaging cholesterol and other fats (called lipoproteins) and carries them through the bloodstream. ApoE composes Very-LowDensity Lipoproteins (VLDLs), Intermediate-Density Lipoproteins (IDLs), and High-Density Lipoproteins (HDL) with triglyceride and cholesterol and plays a key role in the metabolism of these lipoproteins in the blood. Abnormalities in apoE function are related to cardiovascular, neurological, and renal diseases [7]. Glomerulosclerosis, due to the mechanism of atherosclerosis by LDL cholesterol, is one of the renal disease complications. LDL per se induces further production of proteoglycans by vascular smooth muscle cells and retains more LDL in the arterial wall and consequently builds up plaque inside arteries (lipid deposition) which is known as atherosclerosis and ape is an essential protein to transport cholesterol and maintain its homeostasis [7].

Extracellular Vehicles (EVs) such as exosomes and microvesicles are lipid bilayer-delimited particles that are released from the surface of the most cells as a result of various biological processes. Appearance of these vesicles in blood could be the biomarker of renal disease. In the kidney, they released from blood cells, tubular epithelial cells and can be detected in the blood or urine. Microvesicles, as EVs, have been identified in different syndromes such as haemolytic uraemic syndrome that is a condition which affect blood and its vessels and results in kidney failure due to damage to the small blood vessels of kidney, or atypical haemolytic uraemic syndrome that causes abnormal blood clots in the small vessels of the kidney and could restrict blood flow and lead to kidney failure [8]. Renal disease and ESRD are also risk factors for renal cell carcinoma and prolong dialysis increase the risk of renal cell carcinoma and its prevalence is more in men when compared with women [5].

\section{Lifestyle and Role of Physical Activity on Kidney}

Sedentary lifestyle due to the considerable technological revolution is become one of the key effective factors on worldwide epidemic of chronic diseases. In this industrial world, leading the 
physical inactivity is becoming a significant public health issue and link to wide variety of chronic health conditions such as cardiovascular disease, hypertension, or cognitive impairment. Nearly $31 \%$ of adults all over the world are mainly inactive which rises with age and is higher in women than in the men. This unwanted and wide expanded way of living directly and indirectly result in pathogenic disorders such as CKD or ESRD [9-12]. Exercise is an activity which is requiring physical efforts and carried out for specific purpose. Patients with renal diseases show lower levels of physical function which encourage the sedentary lifestyle, disablement process, and muscle impairment. In this occasion, muscle impairment refers to both physiological and structural abnormalities such as muscle atrophy, strength or endurance loss. There are included barriers that increase the level of inactivity such as low confident to pass the exercise or even appear in society, lack of skills, lack of support or time limitation due to their disease. Also, lack of physical activity and exercise knowledge of nephrologist could play considerable role in exapnding sedentary lifestyle amonge CKD patients.

Physical function reduces in early stages of CKD. By progression of disease and expand inactivity, cardiovascular fitness and physical function deteriorate and encourage kidney disease per se $[10,13]$. In stage five of CKD, the physical activity levels drop about $75 \%$ and about half of these individuals unable to do their daily activities while their Vo2max drop to their $17-20 \mathrm{~mL} / \mathrm{kg} / \mathrm{min}$, which means they need to use their $70 \%$ of maximum capacity to carry out normal daily activities [11]. In dialysis patients, taking part in daily physical activity has been proposed that could help and ameliorate dialysis-related symptoms and act as nursing intervention [12]. These programs with interval of two or three times per week which lasted for one hour per session could increase patient's quality of life, independency, strength, and health issues. Recommended and approximate well-studied exercise protocols on renal diseases are those strength and aerobic exercises, while it has been reported diet and proper exercise could control renal failure even in elderly. Performing of active lifestyle instead of inactivity cause beneficial health state for people with renal diseases. Hence, physical activity and exercise improve blood pressure and cardiorespiratory capacity of CKD [12].

Type of exercise is one of the most important research gaps that encourage doing meta-analysis research but generally doing physical activity and exercise help renal disease and CKD patient for having more confident lifestyle by which regular exercise provides health benefits, ameliorate mental health, increase life span, and improve quality of life of all CKD stages $[9,10]$. It is confirmed that regular exercise program is positively associate with walking abilities, better sleep, less bodily pain, and lower BMI. Patients who have regular and even once a week exercise showed lower risk of mortality compared to inactive patient. To explain this mechanism, it has been proposed that the association between exercise regularity and frequency with longer life span is dose dependent that increase weekly exercise sessions that could decrease patients' rate of mortality [9]. Exercise and active lifestyle are irrefutable effective tools to prevent and manage wide range of chronic diseases such CKD and ESRD. It has been reported that doing 20 minutes to 110 minutes physical activity and exercise per session that lasted from two to 18 months could improve physical fitness features such as aerobic capacity, cardiovascular issues such as resting diastolic or systolic blood pressure and heart rate, and nutritional parameters such as albumin or skeletal muscle density. Among all different kinds of training, cardiovascular exercise are highly studied protocols and resistance training which could be combined with cardiovascular exercises are also recommended [14]. Both resistance and aerobic exercise which lasted more than three months (thrice weekly) not only improve cardiorespiratory fitness in renal diseases but increase muscle strength in all CKD stages [11].

There are indirect and direct responsible mechanisms for the diminished physical activities in CKD and renal disease patients. Older age, diabetes, low skill, low self-confidence, and low levels of education are those probable factors of sedentary and inactive lifestyle. Furthermore, anemia or inflammation may play role in this dysfunction. Renal dysfunction-related-physical impairment is independent of age or anemia. In other words, blood oxygen carrying capacity is a factor to determine the capacity of cardiorespiratory fitness. Anemia or low hemoglobin level in the blood is a disorder that put heart under pressure to supply body oxygen needs. It has been shown that despite correction of anemia and improves fitness level of renal disease patients, but it is still below their age matched group. There are some suggested mechanisms that explain the limitation of CKD patients. Autonomic dysfunction, as a situation which Autonomic Nervous System (ANS) cannot work well that affect heart, bladder, or blood vessels, and cause cardiovascular diseases in CKD and renal diseases patients. Endothelial dysfunction is another mechanism to explain renal diseases patients' limitation.

This dysfunction occurs in CKD and evolve kidney disease progression and Nitric Oxide (NO) reduction [9- 11]. NO, as a colorless gas is product of normal endothelium and is a determinant of normal vascular function. It is a compound that widens blood vessels and stimulates release of hormones such as insulin and growth hormone. It has been recognized that CKD patients have insulin resistance and high levels of blood sugar (hyperglycemia) that cause kidney damage. Also, CKD results in GH decrease due to the hyperglycemia which inhibits GH secretion that cause morbidity and mortality rate elevation [15]. Abnormal structure and metabolic muscle function are another explanation for renal diseases patients' limitation. Abnormalities of muscle structure lead to weakness and its-related dysfunction. In CKD and renal diseases, cellular proteins loss and muscle protein catabolism elevate which 
result in muscle weakness. Activation of the Ubiquitin-Proteasome System (UPS), caspase-3, lysosomes, and myostatin are catabolic pathways for losing muscle protein in renal diseases. Surprisingly, although the muscle strength accelerates the following exercise, but it doesn't have any tangible effects on daily physical function. It has been shown that physical inactive people have 5 years shorter life span compare to their same age active people which sedentary lifestyle and physical inactivity is a risk factor for all-chroniccause mortality [9]. In CKD and ESRD, the body skeletal muscles are wasted due to so many reasons. Kidney function disorders leads to uremia and its-related inflammation, hyper-catabolism, oxidative stress, and insulin resistance, which all cause skeletal muscle performance impairment and function loss. Mitochondria, as an organelle in cells are responsible for respiration and energy production. Mitochondria impairment is one of pathological events in CKD and renal diseases.

Emerging oxidative stress, insulin resistance, and lipid metabolism impairment are considered as mitochondria dysfunction indicators. Oxidative stress, as an imbalance between free radicals and antioxidants in body, activates proteolytic pathway which breakdown protein into small polypeptides and cause muscle atrophy. Mitochondria impairment cause its coupling deficiency which consume more oxygen to maintain ATPflux through electron transport chain and consequently increases uncoupling oxidative phosphorylation, plasma isofuran levels (which is eicosanoids formed non-enzymatically by free radical mediated peroxidation of arachidonic acid.), and result in oxidative stress in renal diseases $[13,16]$. In order to know the muscle wasting mechanism, we need to know the role of protein in the body. Proteins are frequently turnover and replace in the cytosol, nucleus, and its related organelles which its rate directly depend on tissues type. Intracellular proteins turnover daily which means, body needs to receive enough protein per day (e.g. protein turnover in a healthy $70 \mathrm{~kg}$ individual is about $280 \mathrm{~g}$ per day) [16]. Proteolysis, which breakdown protein into smaller parts, should be firmly regulated which even small acceleration, contribute to net negative protein balance and negatively alter the cell function and cause muscle loss. Uremic toxin, inflammation, metabolic acidosis and anemia are possible factors that result in muscle wasting $[9,16]$. Metabolic acidosis accelerates protein catabolism, create negative nitrogen balance and consequently cause muscle mass loss in CKD and renal diseases [17].

There are some existing factors that have role in net negative protein balance and muscle wasting in CKD such as decrease in nutrient intake, insulin resistance, chronic inflammation, hormonal abnormalities, hospitalization, and depression [9]. Muscle loss also, occurs in three dimensions; new muscle fibergrowth impairment, protein synthesis-suppression, and protein degradation-acceleration. Muscle fibers and its satellite cells are covered by lamina. In response to changes in growth factor levels (such Insulin-Like Growth Factor 1 known as IGF-1), satellite cells express the myogenic regulatory factors (e.g. myogenin) and started to proliferate and differentiate and finally repair or enlarge myofibrils. In CKD patients, due to decrease in myogenic regulatory factors and IGF-1 impairment, the satellite cells function impaired [16]. IGF-1 regulates muscle protein synthesis. In CKD muscles, protein turnover is affected, and the IGF-1 function is impaired due to the satellite cells dysfunction. In CKD, protein synthesis markers (serine/threonine-protein kinase mTOR and ribosomal protein S6 kinase which is known as p70S6K) decrease and lead to metabolic acidosis and muscle protein loss because of stimulation of muscle protein breakdown (such as leucin) and suppression of muscle protein synthesis.

As a result of CKD-induced protein, catabolism is one of those muscle wasting mechanisms that consequent physical limitations in these patients [16]. There are also mobility assessments that have been published elsewhere and mention its details are far from current review [13]. Glomerular Filtration Rate (GFR) is decreased in renal disease and is also highly associated with muscle impairment [13]. Regular exercises have lots of benefits and aerobic exercises affecting GFR in CKD patients. In early stages of CKD, endothelial function impairs that efferent renal arteriole constrict and per se deteriorate GFR and cause renal vascular diseases.

In response to exercise, blood volume expands which results in renal blood flow elevation and shear stress in kidney. It has been reported that exercise has beneficial in slowing down the CKD progression and could be considered as preventive strategy [18]. Extracellular vesicles that released from lipid layer of cellular membranes, have short half-life (from minutes up to 5 hours) and could transfer receptors and genetic materials and shuttle information to cell. These vesicles have been detected in body fluids such as blood, urine and cerebral spinal fluid. Physiological function of extracellular vesicles included immune modulation, angiogenesis promotion and tissue regeneration. In response to diseases (e.g. inflammation), cellular activation, or cell stress (e.g. exposure to cytokines), the concentration of extracellular vesicles increase and their physiological function (e.g. immune modulation, vessel integrity) changes to pathological and could act as a marker for different types of disorders. Urinary extracellular vesicles and endothelial cell injury are considered as biomarkers of various renal diseases. Extracellular vesicles and exosomes-related disorders and their detailed mechanisms could be finding elsewhere for further study [8]. Muscle mass loss in renal diseases and CKD are considered as main mortality reasons.

Malnutrition is one of those factors that increase muscle wasting. Assessment of muscle mass and its function could be used to determine protein-energy nutritional status [11]. It has been reported that hypoalbuminaemia patients have spontaneously restricted protein consumption but malnutrition per se could 
not increase the rate of mortality and reliable diet could help these patients and approximately compensate protein loss [16]. Hemodialysis leads to protein synthesis degradation which temporarily lasted 2 hours following dialysis. Both uremia and hemodialysis activate cellular protein catabolism [16]. Doing resistance training and its combination with aerobic exercise could elicit muscle hypertrophy in muscle wasting of CKD patients. High intensity resistance training could act against catabolic effect. It has been shown that high intensity resistance training with $80 \%$ of one maximum repetition, thrice a week lasted for 12 weeks could significantly increase cross-sectional-area of muscle [11]. Exerciseinduced inhibiting myostatin pathway in CKD and renal diseases and exercise-induced follistatin upregulation could mitigate inflammation-associated muscle protein degradation, improve insulin/IGF-1 sensitivity, and decrease muscle protein breakdown which consequently promote muscle strength and mass [17]. It has been reported that, low intensity regular walking ( 5 times per week) could ameliorate exercise tolerance, control weight, and control blood pressure. Also, doing exercise in renal diseases and hemodialysis patient help them to better tolerate dialysis-related symptoms such as cramping or restless legs [11].

\section{Role of Nutrition in Treatment of Renal Diseases}

Nutrition, a process of providing necessary food for health and growth, is one of those highly intentioned topics in CKD and renal diseases. CKD and renal disease patients mostly do not intake optimal amount of nutrient that prone them to protein malnutrition or protein energy wasting [19]. Protein energy wasting is a metabolic imbalance which is recognized by loss of body protein in ESRD and CKD. Renal disease patients face with protein metabolism imbalance that is happened by multifactorial reasons such as resistance to anabolic hormone such as insulin and growth hormone, hypercatabolic state induced by uremia, and inflammation. Malnutrition results in metabolic acidosis and hormonal dysregulation which encourage kidney disease [17-20]. It had been recommended that daily protein intake for healthy individual is equal to $0.8 \mathrm{~g}$ per kilogram of body weight. More than half of this amount of protein should be supplied by high biologic value foods that include essential amino acids such as fish, dairy products. High biologic value foods' protein generates acidic products that rely on kidney for their excretion. Kidney used bicarbonate to buffered acids and should regenerate/reabsorb it to continue buffering process to achieve a steady acid-base balance. Every day, renal tubules reabsorb about $4500 \mathrm{mEq}$ of bicarbonate and regenerate additional $75 \mathrm{mEq}$ bicarbonate to keep the required acid-base balance. In renal disease, acidic products accumulate and cause kidney dysfunction.

In CKD the capacity of kidney in excretion of acid diminishes and by progression of CKD, the acidosis becomes worse. In this phase, each nephron undergoes compensatory hypertrophy and produces high amount of ammonia to excrete acid in the form of nontoxic salt such as ammonium. The ratio of ammonia and ammonium lead to accompaniment activation and cause tubule inflammation and fibrosis [17]. Usually, uremic toxins accumulation is associated with renal diseases. Malnutrition associated with oxidation and redox imbalance which are considered as early causes of CKD and renal diseases [19]. The prevalence of protein energy wasting in CKD stages - three to five is about 11-54 percent. It has been shown that uremia-induced alterations could cause energy expenditure elevation, inflammation, and acidosis. Moreover, the combination of nutritional intake, nutrient loss, poor physical activity, and dialysis affect the protein energy wasting that can be seen in Figure 1 [20]. Figure 1. Conceptual model of protein energy wasting, effective physiological factors, glomerular filtration rate in each stage, and interaction of pathological factors on protein energy wasting [20]. Negative protein balance in CKD and renal diseases is due to activation of multiple cytokine (e.g. TNF, interleukin-1 and interleukin-6) mechanism.

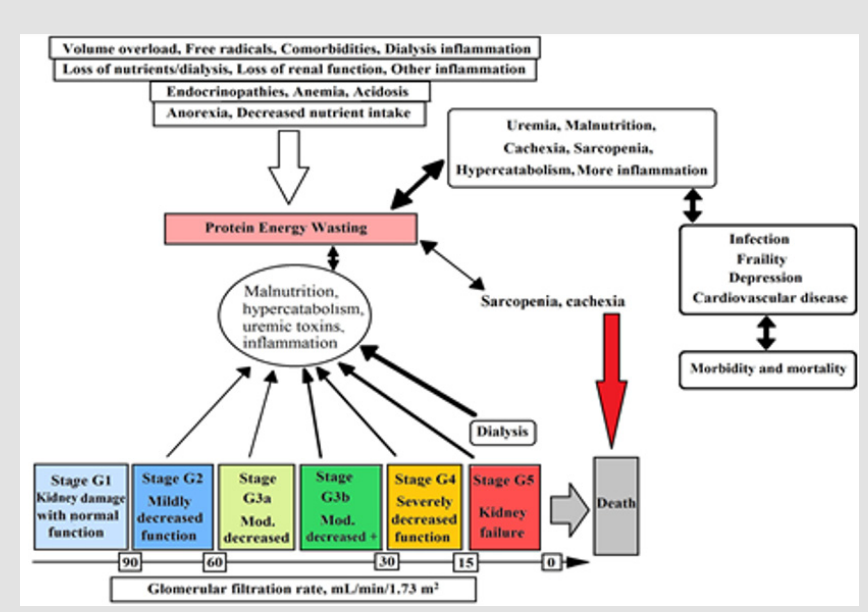

Figure 1: Conceptual model of protein energy wasting, effective physiological factors, glomerular filtration rate in each stage, and interaction of pathological factors on protein energy wasting [20]. 
TNF-related weak inducer of apoptosis which is known as TWEAK associated with regulation pathway of nuclear factor kappa light chain enhancer of activated B cells that is known as NFkappa B. This pathway inhibits myogenesis (formation of muscular tissue) and activates protein degradation cascade. TWEAK and interleukin- 6 are highly expressed in renal diseases patients and are associated with muscle strength loss [17]. In order to diminish the GFR, renal capacity to synthesis ammonia and bicarbonate and excrete hydrogen ion could increase the metabolic acidosis. Clinical definition of metabolic acidosis is the serum bicarbonate concentration that should be less than 22 millimoles per liter. It has been shown that serum levels of bicarbonate acts as a CKD marker which its low level is associated with kidney diseases. This metabolic disorder is so common in stages four and five of CKD while the GFR is between $15-29 \mathrm{~mL} / \mathrm{min} / 1.73 \mathrm{~m} 2$. Correction of metabolic acidosis could reverse all its adverse effects (e.g. muscle protein catabolism, insulin resistance, impaired thyroid hormone) in CKD and renal diseases and slow the progression of kidney diseases [21-22]. Dietary composition is one of the proposed methods to correct acidosis and regain acid-base-balance, muscle mass and even bone metabolism. Usage of animal-derived proteins to supply body essential amino acids generate nonvolatile acidic products which cannot be eliminated by the lungs and could be counterbalanced by the metabolism of base-producing foods such as fruits or vegetables [17].

The alkaline forming plant foods help to neutralize the acidforming protein foods. Alkali therapy can be achieved by using alkaline-rich diet. Although renal diseases patients face with protein energy wasting and malnutrition which is a common metabolic imbalance among them but they cannot have daily protein more than 0.8 gram per kilogram body weight to prevent acidosis and control inflammation, and renal function [20,21]. Acid production from animal protein sources mainly, are a result of methionine and cysteine metabolism, in contrast to bases generate from organic anions such as citrate and malate, which are found in fruit and vegetables. Net acid excretion is 24-hour urinary analysis of ammonium and titratable acid minus bicarbonate which shows strong connection between net acid excretion and $\mathrm{pH}$ value. To examine the food intake, Potential Renal Acid Load (PRAL) is also calculated that indicates the positive value (acid-forming potential) or negative value (base-forming potential). Protein rich foods (cheese, meat, eggs, grain, and animal protein sources) have acid loads with PRAL (mEq/100 g) near 30 and fruit-vegetable foods have alkalizing load with PRAL (mEq/100 g) near minus 20.

PRAL is calculated by selected food items which are presented in Figure 2 (per $100 \mathrm{~g}$ serving) as: PRAL $(\mathrm{mEq} / \mathrm{d})=0.49 \times$ protein $(\mathrm{g} / \mathrm{d})+0.037 \times \mathrm{P}$ or Phosphorus $(\mathrm{mg} / \mathrm{d})-0.021 \times \mathrm{K}$ or potassium $(\mathrm{mg} / \mathrm{d})-0.026 \times \mathrm{Mg}$ or magnesium $(\mathrm{mg} / \mathrm{d})-0.013$ $\times$ Ca or calcium (mg/d) (Figure 2, [21- 22]). Figure 2. Estimated acid-producing potential of selected foods [22]. Energy intake has direct connection with protein catabolism and nitrogen balance. As in negative energy balance, protein started to be catabolized and supply energy which leads to negative nitrogen balance. It has been reported that CKD patients have higher resting expenditure which means that they need to intake higher amount of energy to prevent protein catabolism and negative nitrogen balance and prevent acidosis. Fruit juice is proposed beverage that consisted of citrate. Citrate completely absorbs and metabolized to bicarbonate which consequently could increase $\mathrm{pH}$ of urine (omit acidosis). It has been suggested that due to high energy and the sugar available in fruit juice, individuals only have 0.5 to $1 \mathrm{~L}$ fruit juice per day to prevent subsequent probable side effects such as insulin resistance. Total main purpose of having alkalizing food sources is to increase bicarbonate and help kidney to buffer acidosis. Bicarbonate is a natural element of mineral water which could increase urinary $\mathrm{pH}$ as effective as medical-alkali citrate.

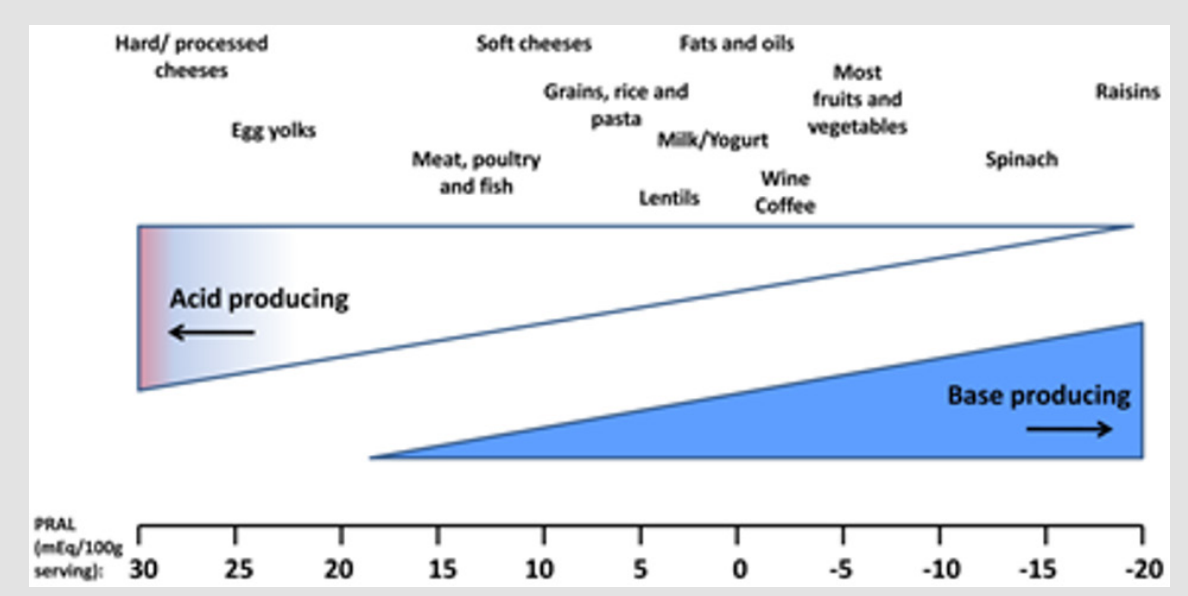

Figure 2: Estimated acid-producing potential of selected foods [22]. 
Alkali therapy can be achieved by both oral supplementation (intake of sodium bicarbonate) and base-producing diet. In the first step, doing dietary modification is a preventive or treatment strategy, as it could control the protein proportion and its subsequent acidosis. In this case, fruits and vegetables need to supply in daily diet and reduce the daily protein intake up to 0.8 gram per body weight $[17,21]$.

\section{Conclusion}

Renal diseases have been found to be one of those highly prevalence disorders that could even cause morbidity and mortality. There are many articles about the different appropriate treatment methods. Among all strategies, doing exercise and control diet are more tangible. Doing exercise improve individual's tolerance to physical activity, help them to control their weight, and control blood pressure as well. Patients with renal diseases and who are under hemodialysis can do exercise which would help them to tolerate dialysis related symptoms/ problems [11]. Nutrition also has irrefutable effects on controlling the balance of acid-base status of kidney. Restriction of some nutrients such as protein has beneficial outcomes for kidney and consuming balance diet (acid-base balance diet), greater intake of fruits and lower intake of animal-related proteins could lower the dietary acid load [22]. In our previous studies, we have studied the effect of food chemicals (cinnamaldehyde, Bisphenol-A and alcohol) induced nephrotoxicity; and have concluded that nutrients / vitamins can protect the kidney from those toxic chemicals [23-26].

\section{References}

1. Levin A, Bilous R, Coresh J (2013) Definition and classification of CKD. Kidney Int Suppl 3: 19-62.

2. NIDDK (2016) Diseases NIoDaDaK. Kidney Disease Statistics for the United States.

3. Chillon JM, Massy ZA, Stengel B (2016) Neurological complications in chronic kidney disease patients. Nephrol Dial Transplant 31(10): 1606 $-1614$.

4. Baluarte JH (2017) Neurological Complications of Renal Disease Seminars in Pediatric Neurology 24(1): 25-32.

5. Tsuzuki T, Iwata H, Murase Y, Takahara T, Ohashi A (2018) Renal tumors in end-stage renal disease: A comprehensive review. Int J Urol 25(9): 780-786.

6. Andrew S Levey LAS, Christopher H Schmid, Yaping Zhang, et al. (2009) A New Equation to Estimate Glomerular Filtration Rate. Ann Intern Med 150(9): 604-612.

7. Saito T, Matsunaga A, Fukunaga M, Nagahama K, Hara S, et al. (2020) Apolipoprotein E-related glomerular disorders. Kidney international 97: 279-288.

8. Karpman D, Stahl AL, Arvidsson I (2017) Extracellular vesicles in renal disease. Nature Reviews -Nephrology 13(9): 545-562.
9. Aucella F, Valente GL, Catizone L (2014) The role of physical activity in the CKD setting. Kidney Blood Press Res 39(2-3): 97-106.

10. Taryana AA, Krishnasamy R, Bohm C, Palmer SC, Wiebe N, et al. (2019) Physical activity for people with chronic kidney disease: an international survey of nephrologist practice patterns and research priorities. 9(12): e032322.

11. Kirkman DL, Edwards DG, Lennon-Edwards S (2014) Exercise as an Adjunct Therapy in Chronic Kidney Disease. Renal Nutr Forum 33(4): $1-8$.

12. Qiu Z, Zheng K, Zhang H, Feng J, Wang L, et al. (2017) Physical Exercise and Patients with Chronic Renal Failure: A Meta-Analysis. Biomed Res Int 2017: 7191826.

13. Roshanravan B, Gamboa J, Wilund K (2017) Exercise and CKD: Skeletal Muscle Dysfunction and Practical Application of Exercise to Prevent and Treat Physical Impairments in CKD. Am J Kidney Dis 69(6): 837-852.

14. Heiwe S, Jacobson SH (2011) Exercise training for adults with chronic kidney disease. The Cochrane Database of Syst Rev (10): Cd003236.

15. Gupta V, Lee M (2012) Growth hormone in chronic renal disease. Indian J Endocrinol Meta 16(2): 195-203.

16. Wang XH, Mitch WE (2014) Mechanisms of muscle wasting in chronic kidney disease. Nat Rev Nephrol 10(9): 504 -516.

17. Zha Y, Qian Q (2017) Protein Nutrition and Malnutrition in CKD and ESRD. Nutrients 9(3): pii E 208.

18. Wyngaert KV, Van Craenenbroeck AH, Van Biesen W, Dhondt A, Tanghe A, et al. (2018) The effects of aerobic exercise on eGFR, blood pressure and V02peak in patients with chronic kidney disease stages 3-4: A systematic review and meta-analysis. MetaAnalysis 13(9): e0203662.

19. Silva RE, Simoes ESAC, Miranda AS, Justino PBI, Brigagao M, et al. (2019) Potential Role of Nutrient Intake and Malnutrition as Predictors of Uremic Oxidative Toxicity in Patients with End-Stage Renal Disease. Oxidative Medicine and Cellular Longevity 2019: 7463412.

20. Hanna RM, Ghobry L, Wassef O, Rhee CM, Kalantar-Zadeh K (2020) A Practical Approach to Nutrition, Protein-Energy Wasting, Sarcopenia, and Cachexia in Patients with Chronic Kidney Disease. Blood Purif 49(12): 202-211.

21. Siener R (2018) Dietary treatment of metabolic acidosis in chronic kidney disease. Nutrients 10(4): piiE512.

22. Scialla JJ, Anderson CA (2013) Dietary acid load: a novel nutritional target in chronic kidney disease? Adv Chronic kidney Dis 20(2): 141149 .

23. Sivakumar JTG, Deveraj H (2006) Effect of food flavour cinnamaldehyde on the antioxidant status of rat kidney. Basic Clin Pharmacol Toxicol 99: 379-382.

24. Sivakumar JTG, Deveraj H (2008) Food flavor cinnamaldehyde - induced biochemical and histological changes in the kidney of male albino wistar rat. Environ Toxicol Pharmacol 26: 68-74.

25. Sivakumar JTG, Kenneth EM (2009) Methods to evaluate the effect of ethanol on the folate analogue, fluorescein methotrexate uptake in human proximal tubular cells. Adv Pharmacol Sci 2009: 6 pages.

26. Sivakumar JTG (2013) An updated review of toxicity of bisphenol A (BPA) with special reference to the kidney. Curr Mol Pharmacol 6(3): 163-172. 
ISSN: 2574-1241

DOI: 10.26717/BJSTR.2020.26.004380

Sivakumar J T Gowder. Biomed J Sci \& Tech Res

(C) (i) This work is licensed under Creative BY Commons Attribution 4.0 License

Submission Link: https://biomedres.us/submit-manuscript.php

\begin{tabular}{ll} 
BIOMEDICAL & Assets of Publishing with us \\
RESEARCHES & - Global archiving of articles \\
\hline ISSN: $2574-1241$ & Immediate, unrestricted online access \\
& - Rigorous Peer Review Process \\
&
\end{tabular}

